\title{
Anti-retro viral therapy adverse drug reaction and associated factors among human immuno deficiency virus infected adult patients at Nigist Eleni Mohammed Memorial hospital, South Ethiopia
}

\author{
Temesgen Tamirat ${ }^{1}$, Kifle Woldemichael ${ }^{2}$, Tsegaye Tewelde ${ }^{2}$, Tariku Laelago ${ }^{3}$
}

1. Wachemo university, Hosanna, Ethiopia.

2. Jimma university, Jimma, Ethiopia.

3. Wachemo university Durame campus, Durame, Ethiopia.

\section{Emails:}

temesgentamirat58@yahoo.com, bethy_kifle@yahoo.com, tsegayetewlde@yahoo.com, tarikulalago@gmail.com

\begin{abstract}
Background: Recent increases in access to HAART have made the management of drug toxicities an increasingly crucial component of HIV care in developing countries. The aim of this study was to determine prevalence of antiretroviral therapy adverse drug reactions and associated factors among HIV-infected adult patients at Nigist Eleni Mohammed memorial hospital.

Methods: A cross sectional study was conducted by retrospective review of patients' medical records. From a total 721 adult patient records, 231 patients record were selected by simple random sampling technique. The study was conducted April15-25, 2015.The association between dependent and independent variables was measured by using OR at 95\% CI. P-value $<0.05$ was considered as statistically significant.

Result: About 53(22.9\%) patients developed ADRs (adverse drug reactions). Female (AOR=2.72, CI=1.177-6.30), patients with WHO stage III and IV $(A O R=13.06, C I=4.17-40.90)$ were found more likely to develop ADRs. Commonly identified ADRs were fatigue (18.1\%), diarrhea (7.7\%), nausea (6.5\%), headache $(3.6 \%)$ and anemia $(2 \%)$.

Conclusion: Nearly one in five patients develop ADRs. Sex of respondents, WHO stage and functional status were associated with ADRs. The health care providers should give due attention to ambulatory, bedridden, and WHO stage III and IV patients.

Keywords: ART; HIV; AIDS; Ethiopia.

DOI: https://doi.org/10.4314/ahs.v20i2.3

Cite as: Tamirat T, Woldemichael $K$, Tewelde T, Laelago T. Anti-retro viral therapy adverse drug reaction and associated factors among human immuno deficiency virus infected adult patients at Nigist Eleni Mohammed Memorial hospital, South Ethiopia. Afri Health Sci. 2020; 20(2): 560-567. https://doi.org/10.4314/abs.v20i2.3
\end{abstract}

\section{Background}

Human immune deficiency virus (HIV) has created an enormous challenge ${ }^{1}$. Globally, an estimated 35.3 million people were living with HIV in $2012^{2}$. An estimated $0.8 \%$ of adults aged $15-49$ years are living with $\mathrm{HIV}^{3}$. Sub-Saharan Africa remains the most severely affected region, with nearly 1 in every 20 adults living with HIV. This accounted for $69 \%$ of the people living with HIV worldwide ${ }^{3}$. In Ethiopia, the overall prevalence of HIV

\section{Corresponding author: \\ Tariku Laelago, \\ Wachemo university Durame \\ campus,Durame, Ethiopia. \\ Email: tarikulalago@gmail.com}

among adults aged $15-49$ was $1.5 \%$ in $2011^{4}$. Ethiopia is among the selected countries that had shown changes in the incidence rate of HIV infection among adults (15-49years old) ${ }^{3}$.

Antiretroviral therapy (ART) prevents people living with HIV from dying from the acquired immune deficiency syndrome (AIDS) and from developing tuberculosis, becoming ill and transmitting tuberculosis and HIV. Emerging science indicates that people should start HIV treatment earlier to realize these benefits ${ }^{2}$. Since 1995, ART has saved 14 million life-years in low and middle-income countries, including 9 million in sub-Saharan Africa ${ }^{3}$. As of December 2012, an estimated 9.7 million people in low and middle-income countries were receiving ART, an increase of 1.6 million over 20112. 
The massive scale up of ART is saving more lives 5 . In 2013, an additional 2.3 million people gained access to the life-saving medicines. This brings the global number of people accessing ART to nearly 13 million by the end of $2013^{6}$. The number of people receiving ART in eastern and southern Africa increased from 625000 in 2005 to approximately 6.3 million in $2012^{7}$. The region accounts for about $84 \%$ of the estimated 7.5 million people who received ART in Africa and $65 \%$ of the estimated 9.7 million people who received ART globally in $2012^{8}$. The number of people receiving ART in Ethiopia increased from less than 9,000 in 2005 to more than 439,000 in $2013^{9}$.

There are different types of ant retro viral (ARV) regimens in Ethiopia. First-line ARV regimens for adults and adolescents in Ethiopia include the following. One of the following should be used unless there are contraindications: The preferred ones include tenofovir disoproxil fumarate (TDF) +emtricitabine (FTC) +efavirenz $(\mathrm{EFV})=$ triple FDC (fixed drug combination). It also comprises ZDV (zidovudine) + 3TC (lamivudine $)+\mathrm{EFV}=$ combivir + EFV. The other preferred options are ZDV+3TC+NVP (nevirapine) $=$ triple FDC.AlternativesincludeD4T (stavudine)/3TC/EFV $=$ double FDC $(\mathrm{d} 4 \mathrm{~T} / 3 \mathrm{TC})+\mathrm{EFV}, \mathrm{TDF} / 3 \mathrm{TC} / \mathrm{NVP}$, $\mathrm{D} 4 \mathrm{~T} / 3 \mathrm{TC} / \mathrm{NVP}=$ triple FDC, ABC (abacavir) /3TC/ $\mathrm{EFV}, \mathrm{ABC} / 3 \mathrm{TC} / \mathrm{NVP}, \mathrm{ABC} / 3 \mathrm{TC} / \mathrm{ZDV}=$ combivir + $\mathrm{ABC}^{10}$.

Like most medicines, ARV drugs can cause side effects. These unwanted effects are often mild, but sometimes they are more serious and can have a major impact on health and quality of life $^{11}$. Recent increases in access to highly active antiretroviral therapy (HAART) have made the management of drug toxicities an increasingly crucial component of HIV care in developing countries. The spectrum of adverse effects related to HAART in developing countries may differ from that in developed countries because of the high prevalence of other conditions such as anemia, malnutrition, and tuberculosis and frequent initial presentation with advanced HIV disease $^{12}$. ART adverse reaction prevalence varies from region to region, country to country ${ }^{1,13,14}$. The severity and profile of ART drug reaction also varies from patient to patients, from drug regimen to regimen ${ }^{14-17}$.

Different studies depicted the ARV regimens and their side effects. Study conducted in Cameron showed that among those who reported ADRs, $29.6 \%$ were on D4T3TC-EFV, $29.3 \%$ on D4T-3TC-NVP, $16 \%$ on AZT3TC-EFV and $10.8 \%$ on AZT-3TC-NVP ${ }^{18}$. Another study done in India conveyed that ZDV+3TC+NVP regimen use reported majority of $\mathrm{ADRs}^{19}$. In Ethiopia, one study displayed that D4T/3TC/NVP was the most commonly used regimen and mostly causes side effects like toxicity ${ }^{20}$.

Continuous evaluation and reporting of unusual effects of ADRs (adverse drug reaction) of ART drug is important for those people receiving ART to get all the help they need to minimize the impact of ADRs. In Ethiopia, there is an ADRs monitoring center. The ADRs monitoring center is responsible for collecting, compiling and analyzing any ADRs information reported by health professionals. Based on this information, risk-benefit evaluations are made and safety measures are taken to protect the public from unnecessary harm. Nevertheless, information on the types and severity of ADRs of ART is inadequate in the study area. Therefore, the aim of this study was to determine the prevalence of ADRs of ART and associated factors among HIV infected adult patients, with the ultimate goal of improving the tolerability and effectiveness of HIV treatment.

\section{Methods \\ Study setting}

The study was conducted in Nigist Eleni Mohamed memorial hospital (NEMMH). NEMMH is found in Hosanna Town, southern Ethiopia. It is located 230kilometers and 194kilometersfrom Addis Ababa and Hawassa, respectively. It renders comprehensive HIV/ AIDS related services including, voluntary counseling and testing (VCT), provider initiated testing and counseling (PITC), prevention mother to child transmission (PMTCT) and ART program. There were721 HIV/AIDS adult patients attending ART in the hospital between 2005 and 2014 .

The patients were initiated on ART based on CD4 cell counts $<200 / \mathrm{mm}$. However, lack of CD 4 count could not preclude ART initiation in patients clinically eligible for treatment. To ensure patient safety, treatment must not be delayed until CD4 count falls below 200. The optimum time to initiate ART is when a patient's CD4 count is 200-350. CD4 count levels do not determine treatment initiation in stage IV patients; regardless of CD 4 count, stage IV patients are promptly started on ART. In stages I and II, immunological assessment is important to initiate ART; in stage III, treatment can be considered under certain clinical conditions with CD4 count of 350 and below. This is a rule in Ethiopia dur- 
ing study period. But currently some modifications are being introduced.

After the $12^{\text {th }}$ week of initiation of ART, patients are scheduled to return every eight weeks. At each visit ARV, drugs and co-trimoxazole preventative therapy for two months are given, counselling of positive living, safe sexual practice, adherence assessment and support are done. Lab tests including alanine aminotransferaseare requested when indicated. CD4 is repeated every 6 months. The ART treatment is given by trained health professional on ART and management of opportunistic infections. The treatment is given in separate ART treatment room.

\section{Study design}

Cross sectional study was done by using retrospective review of 10 years (2005 to 2014) patients' medical record.

\section{Study population and period}

All randomly selected adult patients ( $>15$ years) who were on ART between 2005 and 2014. The study was conducted April 15-25, 2015.

\section{Sample size determination and sampling tech- nique}

Sample size was determined by using a single population proportion formula; considering $5 \%$ margin of error, $95 \%$, level of confidence and $65.5 \%$, prevalence of ADRs among Adult HIV/AIDS(acquired immune deficiency syndrome) patients on ART at the ART clinic of Jimma University Specialized Hospital in $2012^{14}$. After correcting for finite population, 231 samples were included. About 721 registered patients who fulfilled the inclusion criteria were identified, then sampling frame was arranged (1 to 721), finally by applying simple random sampling technique (computer generated method), 231 records were selected.

\section{Data collection, measurements and definitions}

The data were collected using a checklist. Records review covered patients' information from beginning of ART service to their last visit. Data were collected by 3 trained data collectors. The principal investigator and supervisors made a day to day on site supervision during the period of data collection and checked each checklist daily for its completeness and consistency. Both the data collectors and supervisors were trained on the objective, methodology and data collection approach. Data collection format/check list was checked and necessary modification was done before data collection.

Data collected on socio-demographic factors included age, marital status, sex, religion, occupational status, educational status, and initial weight. On clinical and behavioral state; drinking history, khat chewing, WHO stage, initial CD4 count, and functional status were collected. Data on the ART regimen, initial regimen and regimen change; types and frequency of ARVdrugs and ADRs were also collected.

ADRs were defined as unintended and noxious (harmful) response that occurs at normal doses of the drug used for prophylaxis, diagnosis and treatment of diseases. Symptoms reported by the participants, as well as laboratory abnormalities were defined as ADRs while patients are on ART after $6^{\text {th }}$ months follow up ${ }^{12,14}$.

A side effect is the weak form of the adverse effect, which is unpleasant but generally acceptable. The marked changes in dosage schedule or drug withdrawal are usually not necessary ${ }^{12,14,21}$.

Severity of ADRs: those individuals with one drug changed and regimen changed are considered due to the severity of ADRs. Severity can cause life threatening hospitalization result permanent harm/disability or discontinuation of regimen or change in regimen.

Prevalence of ADRs: indicates cumulative prevalence, which indicates patient whoever had an ADR in the past, and switched.

\section{Data processing and analysis}

Data were checked, cleaned, and entered in to Epi data version 3.1, then exported to SPSS version 16 for analysis. The prevalence of ADRs was estimated by using simple descriptive summary statistics such as frequency and proportion. Tables and graphs were used to present the result of the analyzed data. Bivariate analysis was employed to identify candidate variables for further analysis in multivariable analysis. Variables with P-value $<0.25$ in bivariate analysis were transferred to multivariable logistic regression. The association between dependent and independent variables was measured by using OR at 95\% CI. P-value $<0.05$ was considered as statistically significant.

\section{Ethical approval and consent to participant}

Ethical clearance was obtained from Jimma University, College of public health and medical sciences ethical review committee. Permission was obtained first from Hadiya zone health department and NEMMH medical director office. Data were handled confidentially during all phases of research activities. 


\section{Result}

\section{Socio-demographic characteristics}

In this study, a total of 231 patients 'records were reviewed. Of the 231 records, 82(35.5\%) were males and $149(64.5 \%)$ were females. The age range was 15-49yearswith mean of 31(SD 7.63). Concerning marital status, $173(74.9 \%)$ were married and 16(6.9\%) were divorced. Majority, 104(45\%) of the females were housewives. About, 96(41.6\%) patients attended primary school. Most of respondents, 104(45\%) were "protestant religion "followers. For $113(48.9 \%)$ patients, the initial weight was greater than $50 \mathrm{~kg}$ (Table 1).

Table 1: Socio-demographic characteristics of the respondents in NEMMH, Hosanna.

\begin{tabular}{|c|c|c|}
\hline Variables & Total & $\%$ \\
\hline \multicolumn{3}{|l|}{ Age $(n=231)$} \\
\hline $15-19$ & 6 & 2.6 \\
\hline $20-24$ & 30 & 12.99 \\
\hline $25-29$ & 60 & 25.98 \\
\hline $30-34$ & 64 & 27.7 \\
\hline $35-39$ & 32 & 13.86 \\
\hline $40-44$ & 23 & 9.95 \\
\hline $45-49$ & 16 & 6.92 \\
\hline \multicolumn{3}{|l|}{ Marital status(n=231) } \\
\hline Single & 23 & 10 \\
\hline Married & 173 & 74.9 \\
\hline Divorced & 16 & 6.9 \\
\hline Widowed & 19 & 8.2 \\
\hline \multicolumn{3}{|l|}{$\operatorname{Sex}(n=231)$} \\
\hline Males & 82 & 35.5 \\
\hline Females & 149 & 64.5 \\
\hline \multicolumn{3}{|l|}{ Religion(n=231) } \\
\hline Orthodox & 88 & 38.1 \\
\hline Muslim & 36 & 15.6 \\
\hline Protestant & 104 & 45 \\
\hline Catholic & 2 & 0.9 \\
\hline Others & 1 & 0.4 \\
\hline \multicolumn{3}{|l|}{ Occupation(n=231) } \\
\hline Housewives & 104 & 45 \\
\hline Merchants & 31 & 13.4 \\
\hline Government employee & 39 & 16.9 \\
\hline Self employed & 20 & 8.7 \\
\hline Farmers & 21 & 9.1 \\
\hline Students & 15 & 6.5 \\
\hline Unemployed & 1 & 0.4 \\
\hline \multicolumn{3}{|c|}{ Educational status(n=231) } \\
\hline No formal education & 69 & 29.9 \\
\hline Primary education & 96 & 41.6 \\
\hline Secondary education & 57 & 24.7 \\
\hline Tertiary education & 9 & 3.9 \\
\hline \multicolumn{3}{|c|}{ Initial weight in $\mathrm{KG}(\mathrm{n}=231)$} \\
\hline$<40 \mathrm{~kg}$ & 27 & 11.7 \\
\hline $40-45 \mathrm{~kg}$ & 45 & 19.5 \\
\hline $46-50 \mathrm{~kg}$ & 46 & 19.9 \\
\hline$>50 \mathrm{~kg}$ & 113 & 48.9 \\
\hline
\end{tabular}

Clinical and behavioral state at the beginning of ART

Among the selected patients, more than $50 \%$ had started ARV at WHO stage III. Regarding initial CD4 count, more than $94 \%$ patients started ARV with less than or equal to $350 \mathrm{CD} 4$ count. About, 17 (7.4\%) patients had alcohol drinking and khat chewing history. Only 1 person $(0.4 \%)$ had history of cigarettes smoking. The functional status showed that 104(45\%) of patients were able to work (Table 2). 
Table 2: Clinical and behavioral state at the beginning at NEMMH, Hosanna.

\begin{tabular}{|l|c|c|}
\hline Variables & Total & $\%$ \\
\hline Alcohol drinking history & & \\
\hline Yes & 17 & 7.4 \\
\hline No & 214 & 92.6 \\
\hline Khat chewing history & & \\
\hline Yes & 17 & 7.4 \\
\hline No & 214 & 92.6 \\
\hline Cigarettes smoking history & & \\
\hline Yes & 1 & 0.4 \\
\hline No & 230 & 99.6 \\
\hline WHO & & \\
\hline I & 13 & 5.6 \\
\hline II & 76 & 32.9 \\
\hline III & 120 & 51.9 \\
\hline IV & 22 & 9.5 \\
\hline Initial CD4 count & & \\
\hline$=<350$ & 219 & 94.8 \\
\hline$>350$ & 12 & 5.2 \\
\hline Functional status(n=231 & & 45 \\
\hline Working & 104 & 32.9 \\
\hline Ambulatory & 76 & 22.1 \\
\hline Bed ridden & 51 & \\
\hline & & \\
\hline
\end{tabular}

\section{ART drug regimens}

The initial regimen was D4T/3TC/NVP for 123(53.2 $\%), \mathrm{AZT} / 3 \mathrm{TC} / \mathrm{NVP}$ for 49(21.2\%), AZT/3TC/EFV for $10(4.4 \%)$ and other ART for $49(21.2 \%)$ patients. However, only $81(35.1 \%)$ patients were on initial regimen during study period but 150(64.9\%) were not. From those who had changed the regimen, total replaced by other regimen was147 (63.6\%) and only one drug changed was $3(1.3 \%)$.

\section{Drugs used other than ART}

Only $31(23.4 \%)$ patients did not have history of taking drugs other than ART. From those who took drug other drugs other than ART, 196(84.8\%) patients were on cotrimoxazole and 9(3.9) \% patients were on isoniazid prophylaxis.

\section{Types and frequencies of ADRs}

About 53(22.9\%) patients developed ADRs. Therefore, the prevalence of ADRs in NEMMH was $22.9 \%$. For the total ADRs, starting with D4T/3TC/NVP contributed $57.4 \%$. About $20 \%$ of the patients changed the regimen due to AZT/3TC/EFV. Fatigue was common ADRs among initial drug regimen and regimen changed patients (Table 3).

Table 3: Types of adverse drug reactions and regimens at NEMMH, Hossana.

\begin{tabular}{|c|c|c|c|c|c|c|c|c|c|c|}
\hline \multirow[b]{2}{*}{ Variable } & \multicolumn{4}{|l|}{ Initial regimens } & \multirow[b]{2}{*}{$\begin{array}{l}\text { Total } \\
(\%) \\
\mathrm{N}=108\end{array}$} & \multicolumn{4}{|c|}{ Current regimens } & \multirow[b]{2}{*}{$\begin{array}{l}\text { Total } \\
(\%) \\
N=40\end{array}$} \\
\hline & D4T/3TC/NVP & AZT/3TC/NVP & $\mathrm{AZT} / 3 \mathrm{TC} / \mathrm{EFV}$ & Other & & $\mathrm{AZT} / 3 \mathrm{TC} / \mathrm{NVP}$ & ZDV/3TC/NVP & $\mathrm{AZT} / 3 \mathrm{TC} / \mathrm{EFV}$ & Others & \\
\hline \multicolumn{11}{|l|}{ ADRs } \\
\hline Fatigue & 35 & 6 & 1 & 3 & $45(41.7)$ & 0 & 13 & 6 & 4 & $23(57.5)$ \\
\hline Diarrhea & 8 & 4 & 3 & 4 & $19(17.6)$ & 1 & 3 & 0 & 3 & $7(17.5)$ \\
\hline Nausea & 9 & 3 & 1 & 3 & $16(14.8)$ & 2 & 1 & 0 & 2 & $5(12.5)$ \\
\hline Headache & 4 & 4 & 0 & 1 & $9(8.3)$ & 0 & 0 & 1 & 1 & $2(5.0)$ \\
\hline Rash & 2 & 2 & 4 & 0 & $8(7.4)$ & 0 & 0 & 0 & 0 & $1(2.5)$ \\
\hline Vomiting & 2 & 0 & 1 & 1 & $4(3.7)$ & 0 & 0 & 0 & 0 & 0.0 \\
\hline Anemia & 1 & 1 & 1 & 2 & $5(4.6)$ & 0 & 0 & 1 & 1 & $2(5.0)$ \\
\hline $\begin{array}{l}\text { Peripheral } \\
\text { neuropathy }\end{array}$ & 1 & 1 & 0 & 0 & $2(1.8)$ & 0 & 0 & 0 & 0 & 0.0 \\
\hline $\begin{array}{l}\text { Total no } \\
(\%)\end{array}$ & $62(57.4)$ & $21(19.4)$ & $11(10.2)$ & $14(13)$ & $108(100)$ & $3(7.5)$ & $17(42.5)$ & $8(2)$ & $11(27.5)$ & $40(100)$ \\
\hline
\end{tabular}


Factors associated with adverse drug reaction

Variables that were significantly associated at bivariate analysis with $\mathrm{P}$-value $<0.25$ were further examined in multivariable logistic regression to see their relative effect on ADRs.Variables that had an association on bivariate association was transferred to multivariable logistic regression. In multivariable logistic regression analysis sex, functional status of patients and WHO stage were significantly associated with ADRs.

The finding from multivariable analysis revealed that females were 3 times more likely to develop ADRs than males $(A O R=2.72, C I=1.18-6.29)$. Regarding clinical and immunological factors, WHO stage and functional status of patients were significantly associated with ADRs. Patients with WHO stage III\&IV were 13 times more likely to develop ADRs than WHO stage I $\& \mathrm{II}(\mathrm{AOR}=13.06, \mathrm{CI}=4.17-40.90)$. Ambulatory patients were 3 times more likely to develop ADRs than patients who can work $(\mathrm{AOR}=3.06, \mathrm{CI}=1.29-7.29)$. Bedridden patients were 18 times more likely to develop ADRs than patients who can work $(\mathrm{AOR}=18.00, \mathrm{CI}=6.07$ 53.43) (Table 4).

Table 4: Factors associate with adverse drug reaction in NEMMH, Hossana.

\begin{tabular}{|l|l|l|l|l|l|}
\hline Variables & \multicolumn{5}{|c|}{ ADRs } \\
\hline & & & Bivariate & Multivariable & \\
\hline Sex & Yes (\%) & No (\%) & COR at 95\% CI & AOR at 95\%CI & P-value \\
\hline Female & $42(79.2)$ & $107(60.1)$ & $2.53(1.22-5.25)$ & $2.72(1.18-6.29)$ & 0.019 \\
\hline Male & $11(20.8)$ & $71(39.9)$ & 1 & 1 & \\
\hline WHO stage & & & & & \\
\hline III\&IV & $49(92.5)$ & $93(52.2)$ & $11.19(3.88,32.34)$ & $13.06(4.17-40.90)$ & 0.000 \\
\hline I\&II & $4(7.5)$ & $85(47.8)$ & 1 & 1 & \\
\hline Functional status & & & & & \\
\hline Bedridden & $26(47.2)$ & $26(14.6)$ & $15.71(5.83-42.28)$ & $18.00(6.07-53.43)$ & 0.000 \\
\hline Ambulatory & $22(41.5)$ & $54(30.3)$ & $2.36(1.13-4.94)$ & $3.06(1.29-7.29)$ & 0.011 \\
\hline Working & $6(11.3)$ & $98(55.1)$ & 1 & 1 & \\
\hline
\end{tabular}

\section{Discussion}

The prevalence of ADRs among adult HIV/AIDS patients was $22.9 \%$. This is similar to findings of a study done in Zewditu Memorial Hospital, which showed a prevalence of $24 \%{ }^{21}$. However, it is higher than figures from a study in Ghana, which showed the prevalence of ADRs $9.4 \%{ }^{22}$. The difference may be due to differences in recruiting study subjects, as the former study included all patients who were on ART. This can decrease the prevalence of ADRs. The prevalence of ADRs in the current study is also higher than that of a study from Cameroon, which depicted $19.5 \%$ on HAART patients, reported ADRs 18.ADRs in the current study is three fold higher than the systematic review studies, which showed that the overall incidence of ADRs $6.7 \% \%^{23-24}$.

The ADRs in current study is lower than the finding of Guwahati hospital, India, which was 31\% 19. It is also lower than studies of DebraMarkos and Jimma, which showed $51.4 \%$ and $65.5 \%$ of ADRs cases, respective$1 y^{14,25}$. The lower prevalence of ADRs in the current study might be due to our smaller sample size of long period reviews (2005-2014) and using patient data after
6 months follow up. Using data from the 6th months of enrolment created a chance to incorporate the whole history of ADRs of patients and this might have compromised prevalence of ADRs. The reason for the difference could also be certain ADRs are common at the beginning of treatment, and in addition, ADRs can differ based on toxicity of regimens used.

Regarding socio-demographic variables, only sex of patients showed significant association with the development ADRs. The finding of the current study revealed that females were 3 times more likely to develop ADRs than males. This is consistent with the studies from Tanzania $^{26}$, and Ghana ${ }^{27}$. The reason for sex difference in ADRs might be difference in body mass index and fat composition between males and females.

Regarding clinical and immunological factors, WHO stages and functional status of the patients were significantly associated with ADRs. Patients with baseline WHO stages III and IV were found more likely to develop ADRs than patients of stage I and II. This is similar to findings from an Indian study, which showed that clinical stage III and IV were more likely to develop 
ADRs than clinical stage I and $\mathrm{II}^{28}$. Baseline WHO stage III/IV indicated poor clinical status of the patients in Ahmadabad, Gujarat, India ${ }^{29}$. Nevertheless, the study conducted in Ghana reported that WHO stages were not significantly associated with the development of $\mathrm{ADRs}^{22}$. Poor clinical status of the patients might be the leading factor to ADRs, due to patient's drug intolerance, physiological disturbance, and using drugs other than ARVs to treat other opportunistic infections.

Functional status at initiation of treatment showed significant association with the development of ADRs. Ambulatory and bedridden patients during initiation of treatment were found to have higher risk of developing ADRs than patients who could work. The movement of patients might enhance drug distribution. As a result, the ADRs might decrease.

This study has the following limitations: Wide confidence intervals were observed seen, and this might be due to the small sample size. There was lack of clearcut differences between side effects and ADRs largely because of missing data.

The results of biochemical studies, including the blood $\mathrm{PH}$, bicarbonate and $\mathrm{Co}_{2}$ are essential to establish the diagnosis and determine the cause of ADRs. However, biochemical variables were missed and were not included in this study. Recording quality was poor (poor hand writing, incomplete records). Hence, the interpretation of the finding should be taken into account all these factors.

\section{Conclusion}

The prevalence of ADRs was high. Sex of the patients, functional status and WHO stages of HIV/ AIDS showed significant association with ADRs. Females were more likely develop ADRs than males. WHO stage III and IV patients were more likely to develop ADRs than those with WHO stage I and II. Ambulatory and bedridden patients were more risky to develop ADRs than patents who could work. Commonly identified ADRs included fatigue, diarrhea, nausea and headache. The health care providers should give due attention to ambulatory and bedridden patients, and those in WHO stage III and IV since they are more risky to develop ADRs.

\section{List of abbreviations}

ABC: abacavir; ADRs: Adverse Drug Reactions; AIDS: Acquired Immune deficiency syndrome; 3TC: Lamivudine; ART: Anti-Retroviral Therapy; ARV: Antiretroviral drugs used for the treatment of HIV infection; BMI :Body mass index; D4T: stavudine; EDHS: Ethi- opian demographic health survey; HAART: highly active antiretroviral therapy; EFV; efavirent, FDC: fixed drug combination; FTC : emtricitabine; HIV :Human immune virus; $\mathrm{MOH}$ : ministry of health ;NEMMH :Nigist Eleni Mohamed Memorial Hospital ;NFV: Nelfinavir; NVP : nevirapine; ,PLWHA: People living with HIV and manifestations of AIDS; PMTCT: prevention of mother to child transmission; TDF: tenofovir disoproxil fumarate; SNNPR: South nation nationality and people region; D4T: Stavudine; WHO: World health organization; VCT: Voluntary Counselling and Testing; ZDV; zidovudine.

\section{Acknowledgements}

We would like to extend our gratitude to hospital administrative bodies and data collectors for their valuable contribution in the realization of this study.

\section{References}

1. Federal Ministry of Health. Guidelines for implementation of the antiretroviral therapy programme in Ethiopia. Federal HIV/AIDS Prevention and Control Office. Federal Ministry of Health, 2007.

2. United Nations programs on HIV/AIDs. Report on the global AIDS epidemic.2013.

3. United Nations programs on HIV/AIDs. Report on the global AIDS epidemic.2012.

4. CSA: Ethiopia demographic and health survey. Central Statistical Authority of Ethiopia. Addis Ababa, Ethiopia and Calverton, Maryland, USA; 2011

5. United Nations. Program on HIV/AIDs by the AIDS numbers.2013.

6. United Nations programs on HIV/AIDs gap Report on HIV/AIDS.2013

7. UNAIDS. access to antiretroviral therapy in Africa, status report on progress towards the 2015 targets

8. UNAIDS. Getting to zero HIV in Eastern and Southern Africa, regional report 2013.

9. Assefa Y., Alebachew A., Lera M., Lynen L., Wouters E., Damme WV. Scaling up antiretroviral treatment and improving patient retention in care: lessons from Ethiopia, 2005-2013.

10. Federal HIV/AIDS prevention and control office. Guideline for management of opportunistic infections and antiretroviral treatment in adolescents and adults in Ethiopia. FMOH. 2008

11. Antiretroviral-drugs-side-effects UK, 1986-2014.

12. Ramnath S, Chaguturu SK,Mayer KH, Flanigan TP, Kumarasamy N.Review article on Adverse Effects of Highly Active Antiretroviral Therapy in Developing Countries. Clinical Infectious Diseases ,2007; 45:1093-1101 PubMed. 
13. Chelkeba L, Abdissa G. Assessment of ART adverse reactions and determinants at primary hospital in Ethiopia. International Journal of Basic and Clinical Pharmacology, 2012; 2(2).

14. Teklay G, Legesse B., Legesse M.Adverse Effects and Regimen Switch among Patients on Antiretroviral Treatment in a Resource Limited Setting in Ethiopia. J Pharmacovigilance. 2013; 1: 115.

15. Mehta U, Durrheim DN, Blockman M, Kredo T, Gounden $\mathrm{R}$ et.al.Adverse drug reactions in adult medical inpatients in a South African hospital serving a community with a high HIV/AIDS prevalence: prospective observational study. Br J Clin Pharmacol. 2007; 65(3): 396-406.

16. Av kiran R, Ratan j. Lihite. A study on adverse drug reactions in HIV infected patients at an ART centre of tertiary care hospital in Guwahati, India. Asian J Pharm Clin Res, 2013; 6(2): 102-104.

17. Meseret W, Andualem T, Segewkal H. Antiretroviral Therapy Regimen Change Among HIV/AIDS Patients in Nekemt Hospital: a Primary Care Hospital in Oromia Regional State, Ethiopia. Journal of Applied Pharmacentical Science. 2013; 3 (08):036-040.

18. Luma, HN, Doualla MS, Choukem SP, Temfack E, Ashuntantang G, Joko HA et al. Adverse drug reactions of Highly Active Antiretroviral Therapy (HAART) in HIV infected patients at the General Hospital, Douala, Cameroon: a cross sectional study. The Pan African Medical Journal. 2012:1-7.

19. Reddy AVK, Lihite RJ, Lahkar M, Choudhury U, Baruah SK. A study on adverse drug reactions in HIV infected patients at ART center of tertiary care hospital in Guwahati, India, 2013 Asian J Pharm Clin Res, 2013;1 6( 2):102-104.

20. Assefa D, Hussein N. Reasons for Regimen Change among HIV/AIDS Patients Initiated on First Line Highly Active Antiretroviral Therapy in Fitche Hospital, Oromia, Ethiopia. Advances in Pharmacology and Pharmacy 2014. 2(5): 77-83.

21. Assegid T. Assessment of the prevalence of adverse drug reaction among HIV-infected adults on anti-retroviral therapy and factors associated with it at Zewditu Memorial Hospital. Adis Ababa University; 2007.

22. Margaret L, Abena AQ, Ama E,Ernest K.,Vincent G.,Alfred N. Adverse drug reactions to antiretroviral therapy during the early ART period at tertiary hospital in Ghana. Pan African Medical Journal. 2014; $18: 25 \mathrm{Pu}-$ bMed doi:10.11604/pamj.2014.18.25.3886. PubMed.

23. Lazarou J, Pomeranz BH, Corey PN. Incidence of Adverse Drug Reactions in Hospitalized Patients a Meta-analysis of Prospective Studies. Journal of the American Medical Association 1998; 279(15): 1200-1205.

24. Wiffen P, Gill M, Edwards J, Moore A. Adverse drug reactions in hospital settings: a systematic review of the prospective and retrospective studies. Bandolier Extra, 2002; 1-16.

25. Asrat M, Hailu G. Gedafew M, Tesfa M, Prevalence and Associated Factors of Art Adverse Effect among PLWH on Art in Debra Markos Referral Hospital, North East Ethiopia. Fam Med Sci Res. 2013;3:130.

26. William N, Mwamakimbula R. Prevalence and severity of adverse drug reactions among adult patients using default first line and modified antiretroviral combinations in mbeya region, Tanzania., Muhimbili University of Health and Allied Sciences; 2012

27- Margaret L, Abena AQ, Ama E,Ernest K.,Vincent G.,Alfred N. Adverse drug reactions to antiretroviral therapy during the early art period at tertiary hospital in Ghana. Pan African Medical Journal. 2014; 18:25 PubMed doi:10.11604/pamj.2014.18.25.3886 PubMed .

28. Shet A, Antony J, Arumugam, Kumar Dodderi S .Rodrigues R, DeCosta A. Influence of Adverse Drug Reactions on Treatment Success: Prospective Cohort Analysis of HIV-Infected Individuals Initiating FirstLine Antiretroviral Therapy in India. PLos One.2011; 9(3):e91028.

29. Dishank P, Mira D,A.N.Shah,R.K.Dikshit, Early outcome of second line antiretroviral therapy in treatment-experienced human immunodeficiency virus positive patients.Perspect Clin Res. 2013;4(4):215 -220. PubMed 